\title{
Modular AUV System with Integrated Real-Time Water Quality Analysis
}

\author{
Mike Eichhorn ${ }^{1, *(D)}$, Christoph Ament ${ }^{1,+}$, Marco Jacobi ${ }^{2, \ddagger}$, Torsten Pfuetzenreuter ${ }^{2}$, \\ Divas Karimanzira ${ }^{2}$, Kornelia Bley ${ }^{3, \S}$, Michael Boer ${ }^{3}$ and Henning Wehde 4 (D) \\ 1 Institute for Automation and Systems Engineering, Technische Universität Ilmenau, Helmholtzplatz 5, \\ 98693 Ilmenau, Germany; christoph.ament@informatik.uni-augsburg.de \\ 2 Fraunhofer IOSB-AST, Am Vogelherd 50, 98693 Ilmenau, Germany; mja@atlasmaridan.com (M.J.); \\ torsten.pfuetzenreuter@iosb-ast.fraunhofer.de (T.P.); divas.karimanzira@iosb-ast.fraunhofer.de (D.K.) \\ 3 -4H- JENA Engineering GmbH, Mühlenstraße 126, 07745 Jena, Germany; kornelia.bley@web.de (K.B.); \\ boer@4h-jena.de (M.B.) \\ 4 Institute of Marine Research, Nordnesgaten 50, 5005 Bergen, Norway; henningw@imr.no \\ * Correspondence: mike.eichhorn@tu-ilmenau.de or mike.eichhorn@ieee.org; Tel.: +49-3677-69-4103 \\ + The author was with the Institute for Automation and Systems Engineering for the main part of this work. \\ He is now with University of Augsburg, Faculty of Applied Computer Science. \\ $\ddagger \quad$ The author was with the Fraunhofer IOSB-AST. He is now with ATLAS Maridan ApS. \\ $\S$ The author was with the $-4 \mathrm{H}$ - JENA engineering $\mathrm{GmbH}$. She is now retired.
}

Received: 9 May 2018; Accepted: 3 June 2018; Published: 5 June 2018

Abstract: This paper describes the concept, the technical implementation and the practical application of a miniaturized sensor system integrated into an autonomous underwater vehicle (AUV) for real-time acquisition of water quality parameters. The main application field of the presented system is the analysis of the discharge of nitrates into Norwegian fjords near aqua farms. The presented system was developed within the research project SALMON (Sea Water Quality Monitoring and Management) over a three-year period. The development of the sensor system for water quality parameters represented a significant challenge for the research group, as it was to be integrated in the payload unit of the autonomous underwater vehicle in compliance with the underwater environmental conditions. The German company $-4 \mathrm{H}-\mathrm{JENA}$ engineering $\mathrm{GmbH}(4 \mathrm{HJE})$, with experience in optical in situ-detection of nutrients, designed and built the measurement system. As a carrier platform, the remotely operated vehicle (ROV) "CWolf" from Fraunhofer-Institut für Optronik, Systemtechnik und Bildauswertung - Institutsteil Angewandte Systemtechnik (IOSB-AST) modified to an AUV was deployed. The concept presented illustrates how the measurement system can be integrated easily into the vehicle with a minimum of hard- and software technical interfaces.

Keywords: autonomous underwater vehicle; water quality monitoring; in situ-detection of nitrates

\section{Introduction}

Aquaculture production increased from 5 million to 63 million tons during the last three decades, while capture fisheries production increased from 69 million to 93 million tons in the same time period [1]. Studies predict that aquaculture will supply $62 \%$ of fish destined for direct human consumption by 2030 [2]. While forming a great economic success, the ecologic impact of this increase is widely unknown. Furthermore, the industry currently lacks cost efficient observational methodology to face the challenge of determining the environmental consequences which are rooted in intensive livestock breeding with massive concentrations of fish excrements and non-controlled nutrient surpluses. 
The Marine Directive of the European Union is aimed at good environmental status (GES) of the EU's marine waters by 2020 [3]. A sustained and cost-effective monitoring of the water quality within European coastal areas is of growing importance to achieve this goal.

This paper describes an approach to improve the observational methodology of the environmental impacts by developing an automatic, unmanned underwater measuring device for sampling the water column properties. The device measures the standard physical water body properties such as temperature and salinity. Additionally, the system allows for observing other parameters such as oxygen, chlorophyll and nutrients, which are all crucial for the welfare of the naturally living resources near aquaculture sites.

Nowadays, there are many systems for monitoring fish farms. An overview about relevant works for tanks and ponds is presented in [4]. In this paper, a low-cost wireless sensor network for monitoring the water quality and the fish behavior in aquaculture tanks during the feeding process will be proposed. The system monitors the water quality parameters (temperature, conductivity, turbidity, oil layer), the tank state parameters (illumination, water level) and the fish behavior using their depth and the velocity information. The data transfer occurs via WiFi technology. The presented water quality monitoring system in [5] uses ZigBee for communication and the LabVIEW Software (National Instruments, Austin, TX, USA) for online visualization. The real-time data transmission in aquaculture monitoring systems is an important research field in recent years. Requirements like low cost and power consumption, high transmission quality, limited energy capacity, transmission rate and range have to be met. A long lasting underwater wireless sensor network using acoustic modems to solve these challenges was proposed in [6].

All presented systems above allow long-term and stationary monitoring of fish behavior and water parameters. The main issue of these systems is the spatially limited measurement in a fixed depth, where only a few measurement locations can be observed. Moreover, the equipment can stay a long time under water and can provide online data. In combination with a spatially distributed measurement approach using an underwater vehicle like an ROV or an AUV, it is feasible to combine the data and augment the stationary measured values with the vehicle measurements. Furthermore, the long-term deployment of sensors needs to consider the topic of biofouling and maritime growth that can influence the measurements. Some sensors need regular cleaning, where a short-term deployable platform such as an underwater vehicle is a good alternative.

Although several ROV applications in fish farms have been published in the past few years, the use of AUVs to solve possible tasks in and around fish farms is scarce. ROVs are in many cases a cost-effective alternative and less dangerous than using divers. In [7], an accurate navigation and guidance system for an ROV based on an ultra-short baseline (USBL) system and a Doppler Velocity Log (DVL) system was presented to support net pen inspections. This application is also the main issue in [8] where an ROV and an AUV were used. The cleaning of aquaculture nets is a further challenge for ROVs, which is presented in [9]. In [10], an ROV was used to determine the fish size for abalone fishing by using a stereo camera.

This paper includes a summary of the main results of the research project SALMON and has been previously published partly in [11-13]. The objectives of the project were: (i) to develop a miniaturized sensor system for real-time water quality analysis (ii) to assess the prospects for the implementation of such a sensor system in an AUV, and (iii) to examine the possibilities of using an AUV for water quality monitoring around fish farms.

\section{Material and Methods}

\subsection{Hardware}

\subsubsection{AUV Platform}

As a carrier platform for mounting the sensor system, the Hybrid Autonomous Underwater Vehicle CWolf from the Fraunhofer IOSB-AST was used. The vehicle can operate fully 
autonomously or tethered with a fiber optic connection. Its modular payload concept allows for an easy sensor integration. The CWolf hardware is based on the pressure vessel construction of the widely mission tested Seawolf ROV produced by ATLAS ELEKTRONIK, Bremen, Germany.

The pressure vessel construction is segmented as shown in Figure 1. Each segment contains systems for a specific task such as propulsion, navigation, power supply, vehicle guidance or for payload sensors. With this approach, the vehicle can be reconfigured for a required application task.

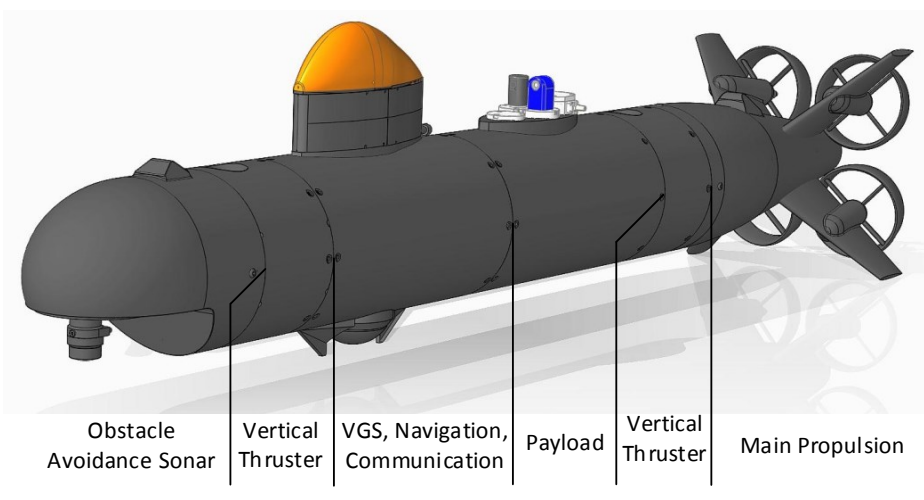

Figure 1. AUV CWolf.

The CWolf is equipped with four stern propellers for movement and maneuvering the vehicle. Different control commands of the horizontal or vertical propeller pairs result in a yaw and pitch torque. This allows the rotation of the vehicle without rudder units. Two additional vertical thrusters at the bow and stern are used for hovering and for pitch control during low speed operation. Due to the modular concept, additional horizontal thrusters can be added so that the vehicle can move sideways. This concept also allows for an easy integration of the application task with a minimum of hardand software technical interfaces. Different kinds of payloads can be integrated into the pressure hull segment. For different payloads, different hull segments can exist and this makes the payload exchange very simple. A flange mechanically connects the segments and a bus system provides standard power supply and communication interfaces such as Ethernet, RS232 and Controller Area Network (CAN). The vehicle base platform provides accurate navigation data for vehicle guidance and geo-referencing the measured values. It consists of an inertial navigation system (INS) using several technologies such as a fiber optic gyro, a Doppler-velocity log, Global Positioning System (GPS), pressure sensors and USBL localization. The most important technical data of the AUV is shown in Table 1.

Table 1. Technical data of AUV CWolf.

\begin{tabular}{cc}
\hline Parameter & Value \\
\hline Length & $2.20 \mathrm{~m}$ \\
Diameter & $0.30 \mathrm{~m}$ \\
Weight in air & $135 \mathrm{~kg}$ \\
Max. speed & $6 \mathrm{kn}$ \\
Endurance at $3 \mathrm{kn}$ & $3 \mathrm{~h}$ \\
Payload & $15 \mathrm{~kg}$ \\
\hline
\end{tabular}

\subsubsection{Sensor System}

The company $-4 \mathrm{H}$ - JENA engineering GmbH (4HJE) (Jena, Germany) [14] developed and manufactured a miniaturized sensor system for the payload unit. Table 2 shows the main parameters of the system. Some of these parameters are easily measurable using standard sensors available on the market. However, the nitrate measurement is a difficult process. Through the limited space conditions in the AUV and the requirement of a low hydrodynamic resistance standard nitrate sensors like 
ISUS (In Situ Ultraviolet Spectrometer) [15] or SUNA (Submersible Ultraviolet Nitrate Analyzer) [16] cannot be used. These systems have a length of over $600 \mathrm{~mm}$ and also accuracy limitations in clouded water [17]. Although there are very accurate wet chemical methods, they are not practical for mobile underwater use. $4 \mathrm{HJE}$ has carried out extensive research on optical in situ-detection of nitrates in earlier projects, expertise that is used in this project. The following section presents a short introduction about the optical measurement principle and a few technical details.

Table 2. Parameters of the sensor system.

\begin{tabular}{cc}
\hline Parameter & Measuring Range/Value \\
\hline Sodium nitrate $\mathrm{NaNO}_{3}$ & $43-2000 \mu \mathrm{g} / \mathrm{L}$ \\
Oxygen concentration $\mathrm{O}_{2}$ & $0-500 \mu \mathrm{mol} / \mathrm{L}$ \\
Conductivity $\sigma$ & $0-75 \mathrm{~ms} / \mathrm{cm}$ \\
Temperature $\mathrm{T}$ & $-5-40^{\circ} \mathrm{C}$ \\
Measurement cycle & $1 \mathrm{~s}\left(\mathrm{O}_{2}, \sigma\right) / 5-10 \mathrm{~s} \mathrm{NaNO} 3$ \\
Power Supply (Computer) & $12 \mathrm{~V}$ \\
Power Supply (Sensors) & $19-25 \mathrm{~V}$ \\
Energy Consumption & $12 \mathrm{~W}(12 \mathrm{~V}) / 10 \mathrm{~W}(22 \mathrm{~V})$ \\
\hline
\end{tabular}

The developed sensor system is integrated in a cylindrical hull segment matched with the diameter of the entire submerged body. Ring-shaped adapters in combination with sealing rings allow a pressure-tight connection to the rest of the submerged body. The off-the-shelf sensors used allow easy maintenance and changing from the outside without removing the segment. In addition, the inlet and outlet filters are changeable during field operations. Figure 2 shows the components and sensors of the sensor system.

The water analysis process follows the following path: Inlet Filter $\rightarrow$ Micro Annular Gear Pump $\rightarrow$ Cuvette Unit $\rightarrow$ Temperature Measurement $\rightarrow$ Outlet with Check Valve and Filter. The Scientific Computer (SC) and the Measurement Computer (MC) are situated in the lower part of the segment. These computers were connected with the internal vehicle communication unit via Ethernet. Since the nitrate measurement water had to be pumped through the vehicle, a leak sensor was installed at the bottom of the hull segment.

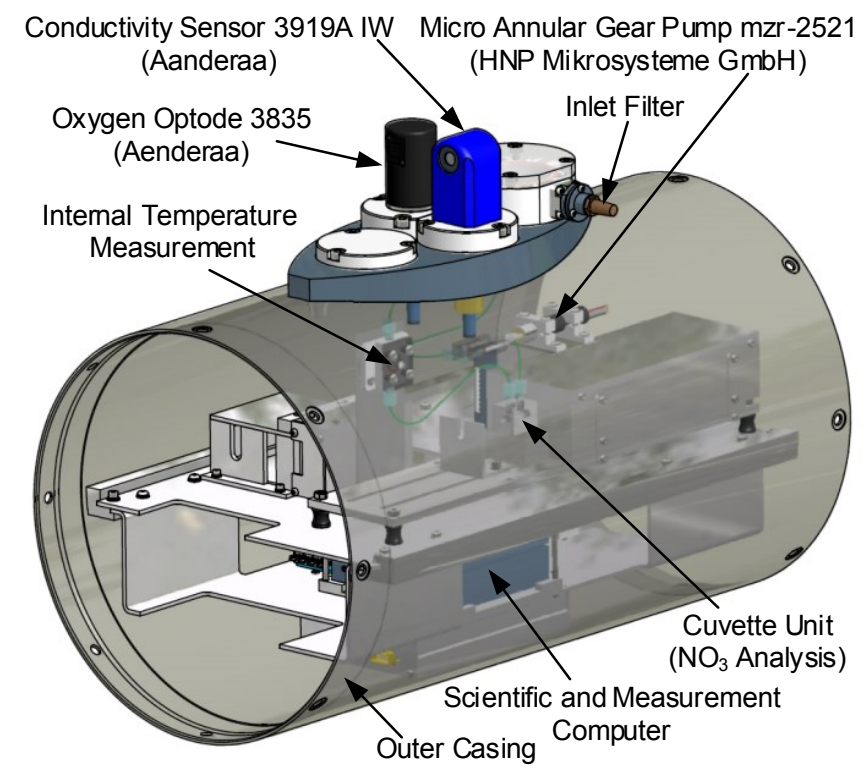

Figure 2. Computer-Aided Design (CAD) drawing of the sensor system (-4H- JENA engineering $\mathrm{GmbH}-4 \mathrm{HJE})$. 


\subsubsection{Nitrate Measurement}

For the determination of nitrate, an optical method is used. The medium to be measured is pumped through a measuring cuvette via a hose system. A deuterium lamp emits ultraviolet radiation at the medium in the cuvette (see Figure 3). Some wavelengths are absorbed stronger than others by nitrate (see Figure 4).

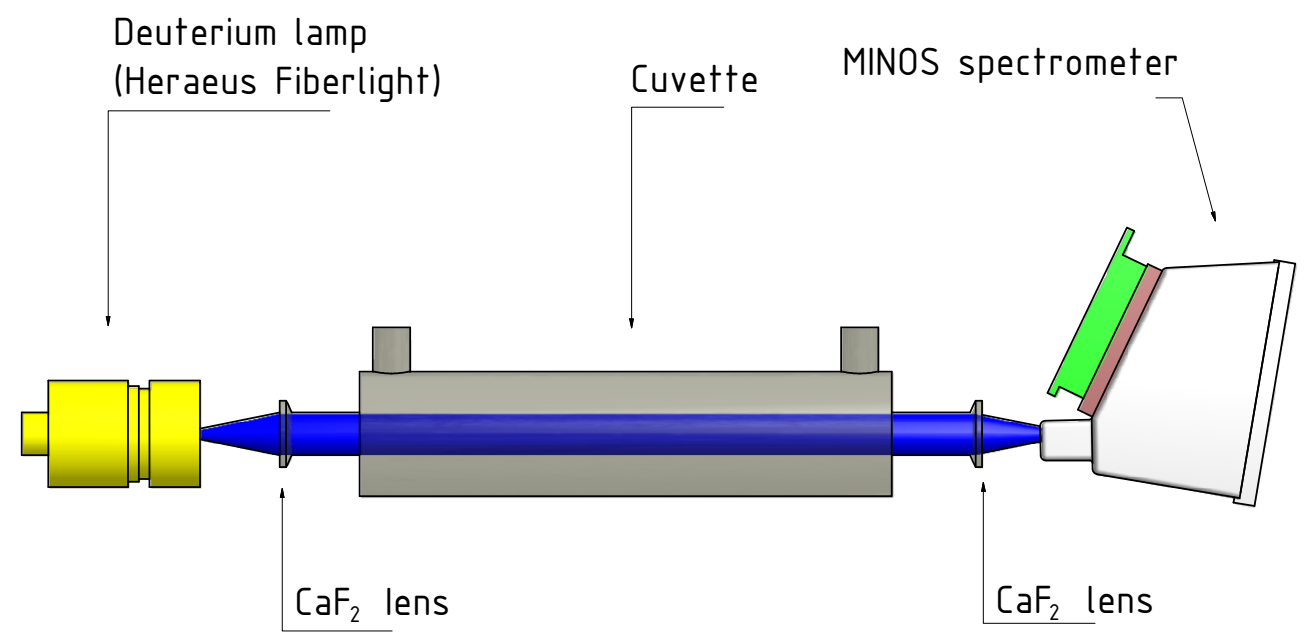

Figure 3. Measuring principle of nitrate determination.

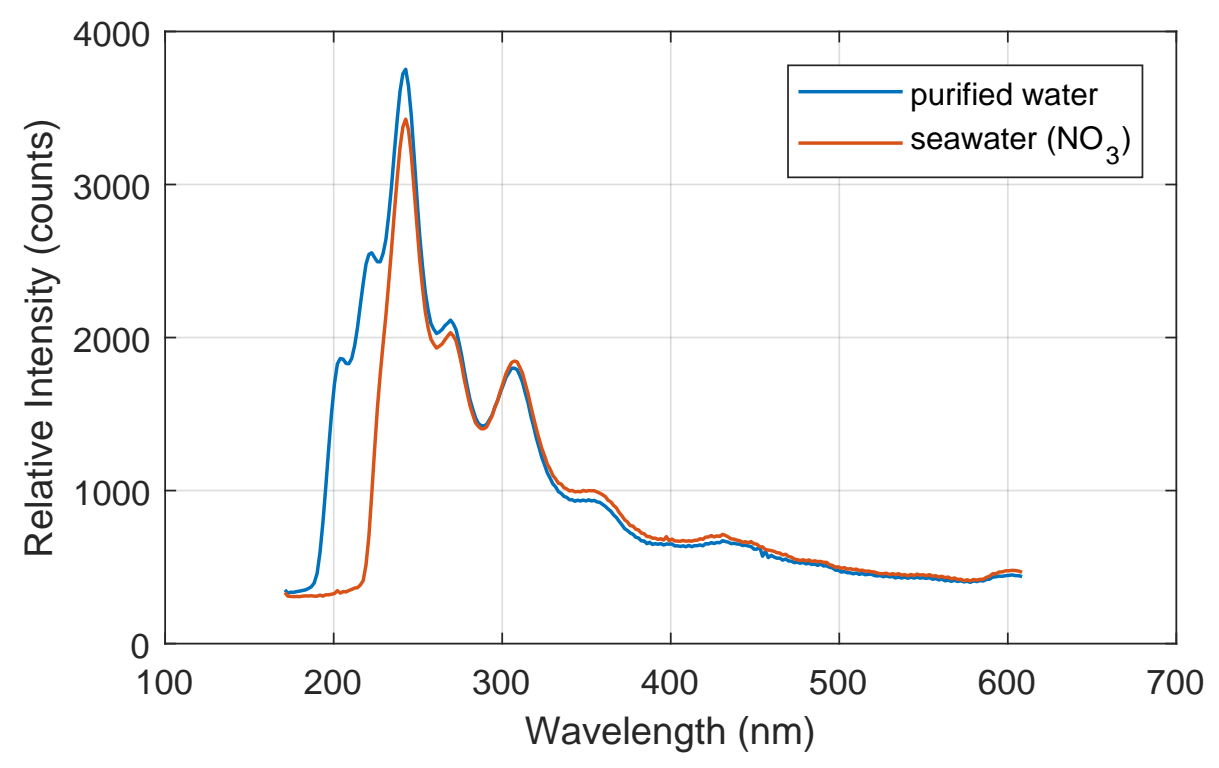

Figure 4. Spectra of purified water and seawater.

The integrated spectrometer detects the intensity of the different wavelengths. Using stored spectra of purified water or seawater with known nitrate concentrations, the current nitrate concentration can be calculated by the MC. Additional factors influencing the measurement are the temperature of the measured medium, as well as other components in seawater. These are considered in the calculations.

To adjust the measuring range of the system, the length of the cuvette must be selected accordingly. In a longer cuvette, more light is absorbed even when the nitrate concentration is low, thus the sensitivity increases with the length of the cuvette. If the cuvette is too long, too much light is absorbed 
and the spectrometer receives too little values for the calculation. The cuvette, which is used in the mobile analyzing unit has a length of $1 \mathrm{~cm}$ and is shown in Figure 5.

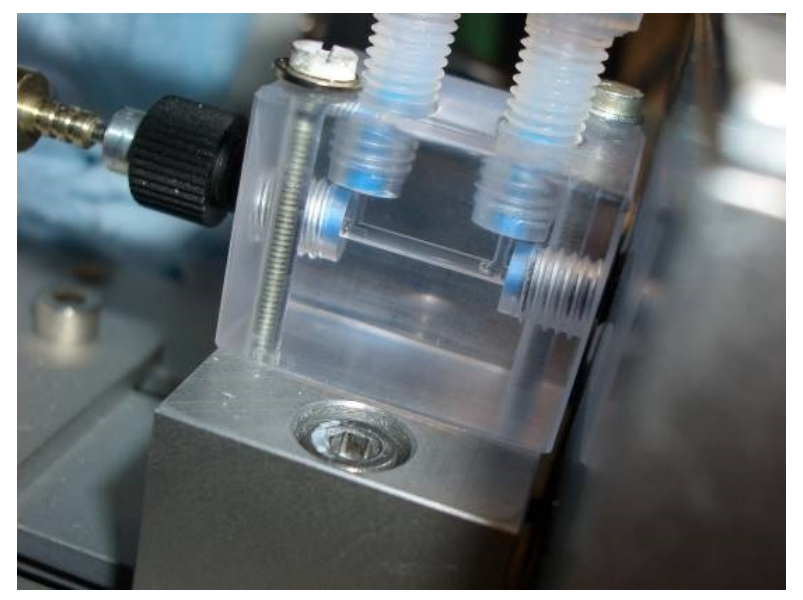

Figure $5.1 \mathrm{~cm}$ cuvette for nitrate measurement.

\subsection{Software}

The modular control software ConSys [18] is responsible for controlling and monitoring the base functionalities of the autonomous vehicle. This software is a development of the Fraunhofer IOSB-AST group and has already been used successfully in various underwater projects of this group.

\subsubsection{Communication Concept}

The algorithms for navigation, control and monitoring the electrical components (power supply, computer equipment, actuators and sensors) run on the Control Computer (CC), which is located on the vehicle base platform. It provides an interface to receive navigation data, the vehicle status and error messages and controls several actuators in the Low Level Controller (LLC). The vehicle guidance runs as a backseat driver on a Scientific Computer (SC), which is located in the payload unit. A Measurement Computer (MC) for the water quality measuring management is also located there. For communication between the applications on the computers, the User Data Protocol (UDP) is used. This ensures operating system and compiler independency of the interacting software programs. Figure 6 shows the interfaces between the programs and the defined message telegrams for the exchange of information/data and for the delivery of control commands.

The telegram LLC_Command is needed to activate the vehicle control from the SC. Therefore, it can be switched to a controlled mode, a direct mode or off mode, when the SC finishes the vehicle control. In controlled mode, the LLC requires a heading, depth and speed command. In the SALMON project, direct mode was used whereby the SC controls the vehicle propulsion engines directly. It has to send a LLC_Setpoints_CWolf telegram, which includes the Pulse-width modulation (PWM)-values for each single motor.

The CC distributes the actual navigation data every $100 \mathrm{~ms}$ to all software modules. This is more than what is used by the SC and MC to handle the mission, to control the vehicle and to record the water quality measurements. The NAV_Data telegram includes the actual vehicle location (latitude, longitude and depth values), the vehicle orientation, speed, and height over ground values. The LLC of the CC communicates between the motor hardware and the CC-software. It receives the LLC_Setpoint_CWolf and LLC_Setpoint telegrams and sends LLC_Status, containing the current motor status, and LLC_Error telegrams, when an error has occurred. The unique identification of telegrams occurs with a header at the beginning of a telegram, which contains a message Identification (ID) and the message-payload length. 


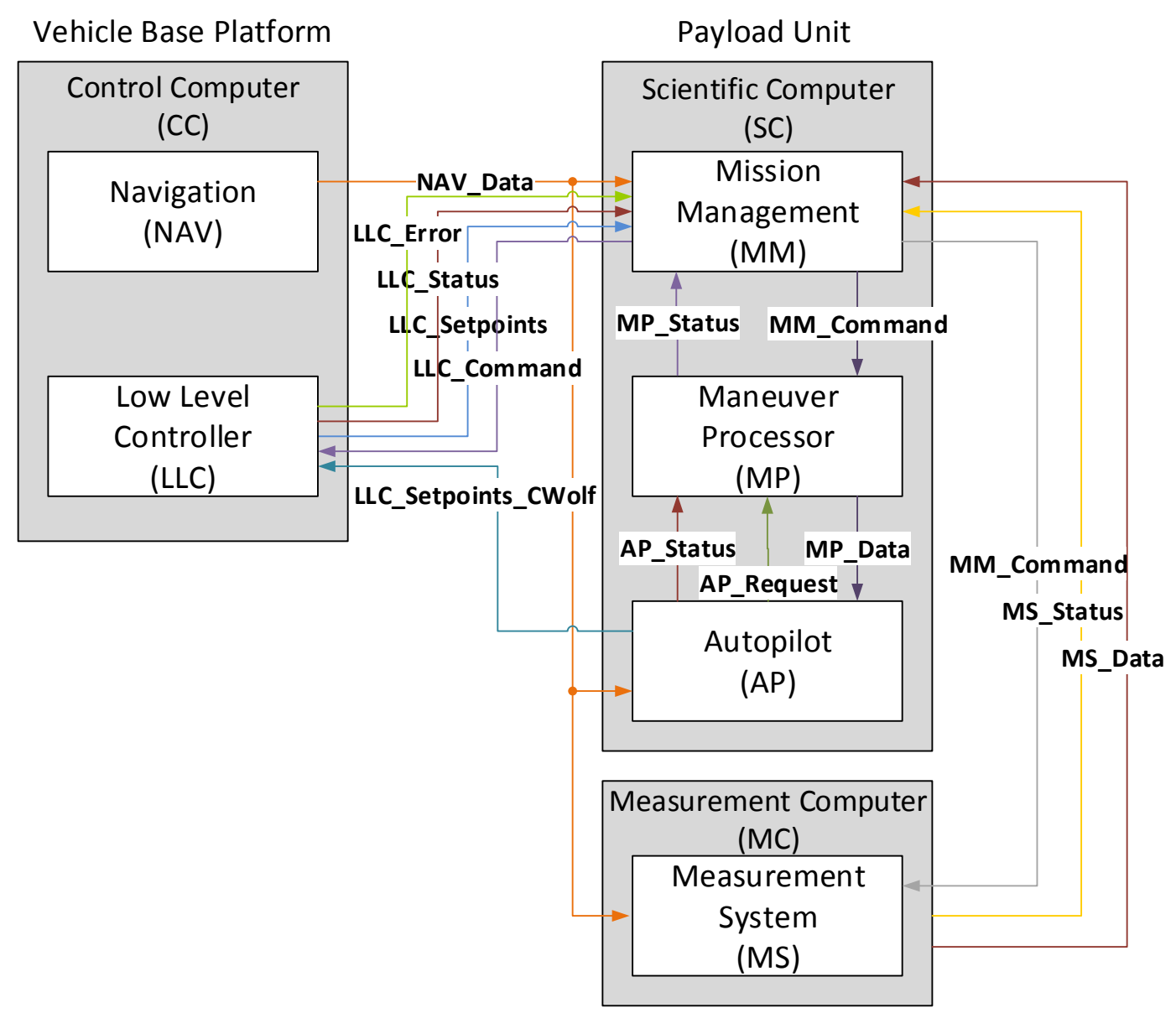

Figure 6. Interfaces and message telegrams between the computers.

\subsubsection{Mission Planning}

To create a mission plan, a menu-guided planning system was used. This system evolved from the planning system developed in the EU project GREX [19] and considers the experiences gathered there. The user will be presented with information and dialogs, which are necessary to solve the actual planning task step by step. The typical planning sequence includes three stages:

(1) Defining the sea chart/area of interest,

(2) Selection of the vehicle, and

(3) Build a plan using defined mission elements.

Figure 7 shows the possible mission elements (vehicle primitives). Available elements include an initial and a final element (to define the start and goal position of the mission), the three base maneuver elements, (waypoint, line and arc) and the complex mission element meander. The configuration of several elements can be started by choosing the respective element in the lower half of the window. The generated mission plan will be stored in a text file. At the beginning of a mission, the Maneuver Processor loads and parses this text file in a map structure to use it during the mission. 


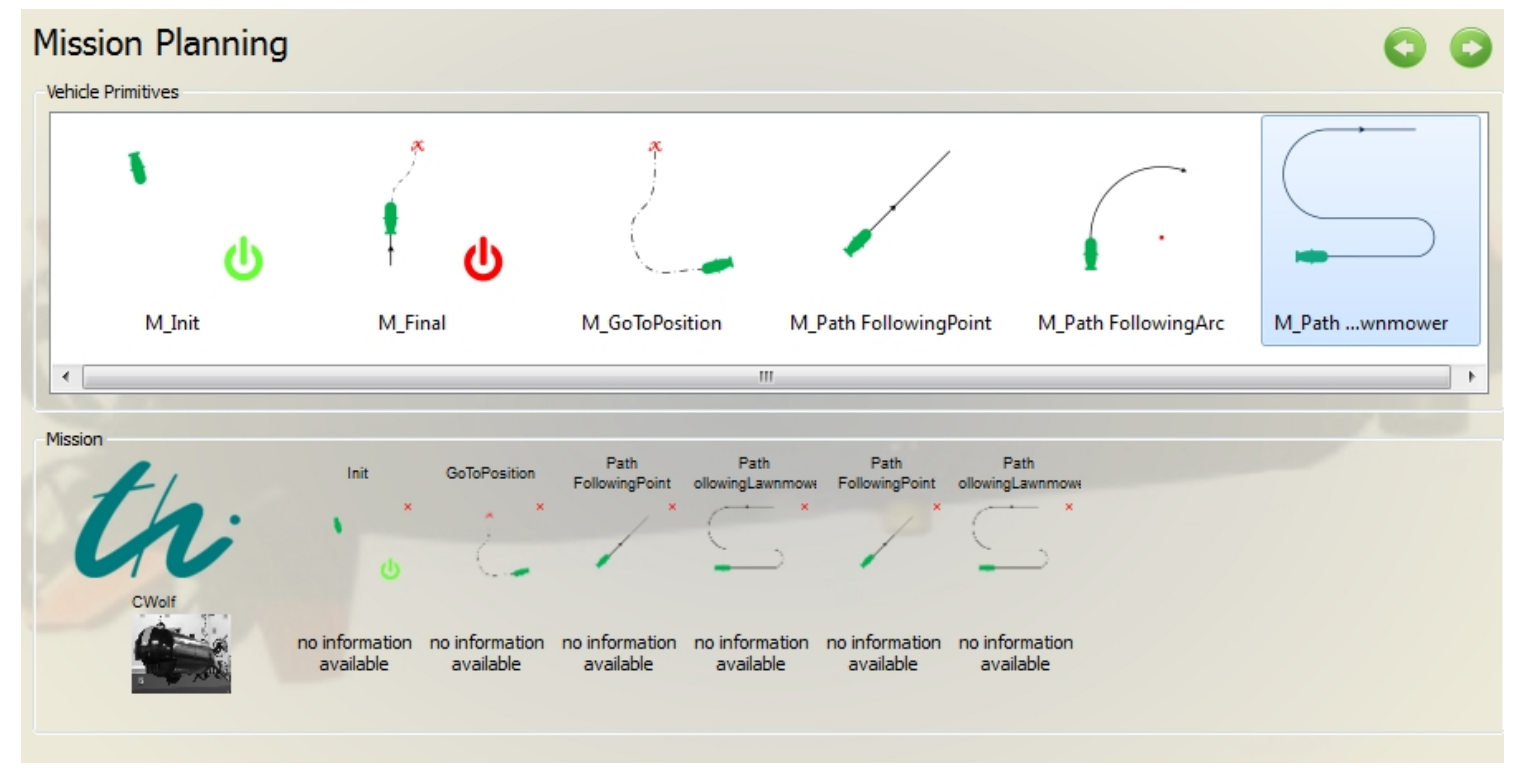

Figure 7. User menu for element-based planning of a mission.

\subsubsection{Dive Profiles}

The dive profiles are yo-yo style and arranged in a meander to cover the whole area. The mission element meander is specified by the following parameters: start position $x_{\text {meander }}, y_{\text {meander }}$, maximum depth $z_{\text {max }}$, rotation $\theta_{\text {meander }}$, leg length $l_{\text {leg }}$, distance between two legs $d_{\text {leg }}$ and the number of legs $n_{\text {legs }}$. Figure 8 shows several parameters to define a waypoint list according to the horizontal path for the meander element. The angle $\alpha_{\text {arc }}$ will be used for the reproduction of the arc element. This also defines the angle differences between adjacent waypoints on the arc. To generate a smooth transition between a line segment and an arc, a waypoint will be positioned before the start and behind the end position of the arc at a distance of $d_{\text {arc }}$. In the figure below, these "arc"-waypoints are signed with an " $x$ " mark.

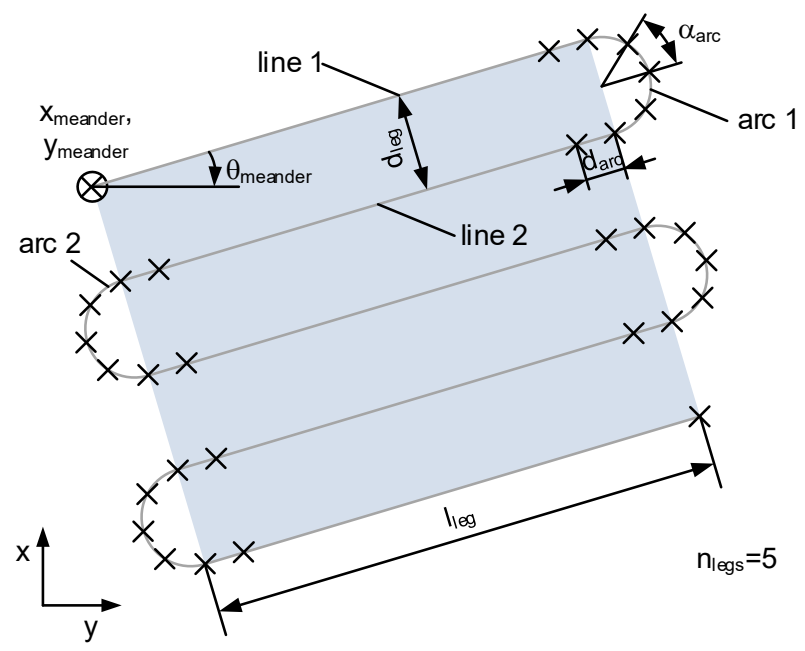

Figure 8. Horizontal parameters of the meander element.

To detect the water quality parameters in several depths, two dive profiles were preferred. The first has a saw tooth shape (see Figure 9a) similar to a Slocum Glider dive profile [20]. This allows the recording of measurement data at every depth in the least amount of time using a minimal number of course changes. Therefore, the area of interest can be recorded in a minimal duration with small energy consumption by the AUV. This dive profile requires fast response times of the sensors in comparison to 
the changes in value of the water quality parameters during the "dive-up" and "climb-to" maneuvers. At the beginning of the mission, the AUV uses the thruster to arrive to the depth $z_{\min }$. After that, the AUV uses the propellers and starts with the diagonal maneuvers. In case of complex vertical changes in the parameters or sensors with large response times, stepwise "dive-up" and "climb-to" maneuvers executed in hover mode can be used. This dive profile requires more energy and examination time for the area of interest in comparison to the saw tooth dive profile. Figure $9 \mathrm{~b}$ shows this defined profile. To support the navigation with actual GPS positions, a surfacing is required when the distance traveled by the underwater vehicle reaches $d_{\text {dive_max }}$.
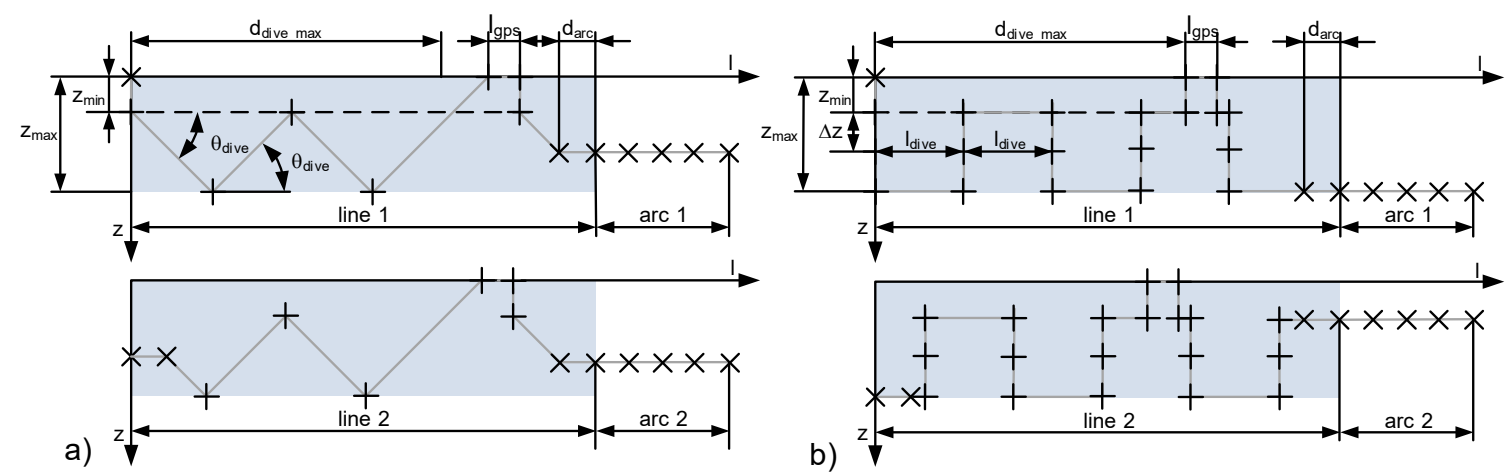

Figure 9. Vertical dive profiles (a) saw tooth profile; (b) square profile.

\section{Results}

\subsection{Test Description}

The field trials of the prototype were carried out in the second half of October 2013. The operation area is located about $30 \mathrm{~km}$ south of Bergen, Norway on the island of Austevoll near the experimental fish farms of the Institute of Marine Research (Figure 10). The goal of the trials was:

(i) to test the autonomous guidance of the vehicle and the sensor system in practice, and

(ii) to monitor the water quality around the fish farms.

To receive the current vehicle position and its status as well as the measurement data online, the vehicle was tethered via a fiber-optic cable also during the autonomous missions. This allowed the manual control of the vehicle in case of an emergency, like an inrush of water or a collision situation with a passing ship.

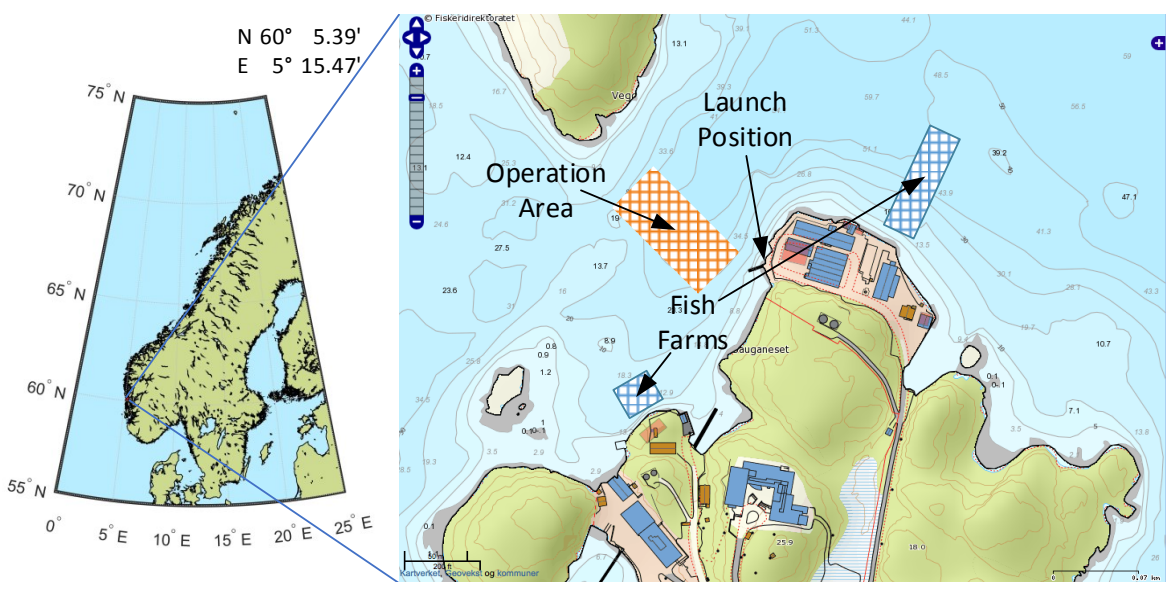

Figure 10. Operation area, Austevoll, Norway. 
The first trial focus included the validation and adaption of the vehicle model and the controller design on the used vehicle structure as well as the tests of the guidance algorithms on the vehicle and the developed mission planning system. During the individual dives, the sensor system was active and logged water quality data. These trials occurred in a sheltered bay without shipping traffic. The access to the water occurred by a ramp using the transport trolley.

The final trials were conducted between the two fish farms (see Figure 10). To launch the vehicle near the operation area, a mobile crane was used (see Figure 11). This requires careful work to avoid an impact of the sensitive sensor system or the propellers with the land stage or the transport trolley.

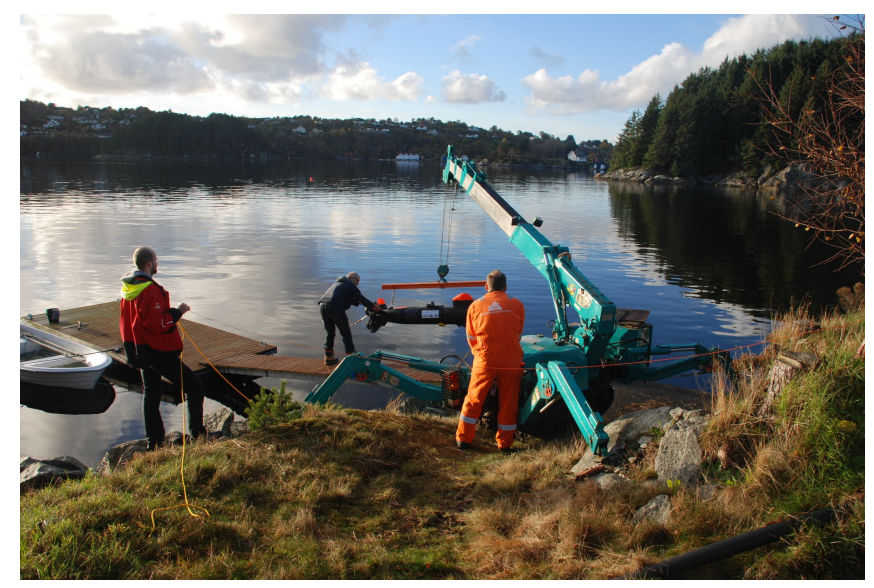

Figure 11. Launching of the vehicle near the fish farms in Austevoll.

For the mission, a meander-shaped plan consisting of 45 waypoints was defined. The farm locations were also considered in the mission planning as 'no go area'. This allows for keeping a safe distance to the fish farms to avoid possible interactions between the AUV and the farms. The AUV headed for the individual waypoints (black marked ' $x$ ' in Figure 12) and dove up to the deepest depth in the bay using a square dive profile, which is presented in Section 2.2.3. The choice of this profile shape results from the limited space in the operation area. The first dive maneuver had to be interrupted, as there was a risk of a cut through the fiber optical cable by a fishing boat.

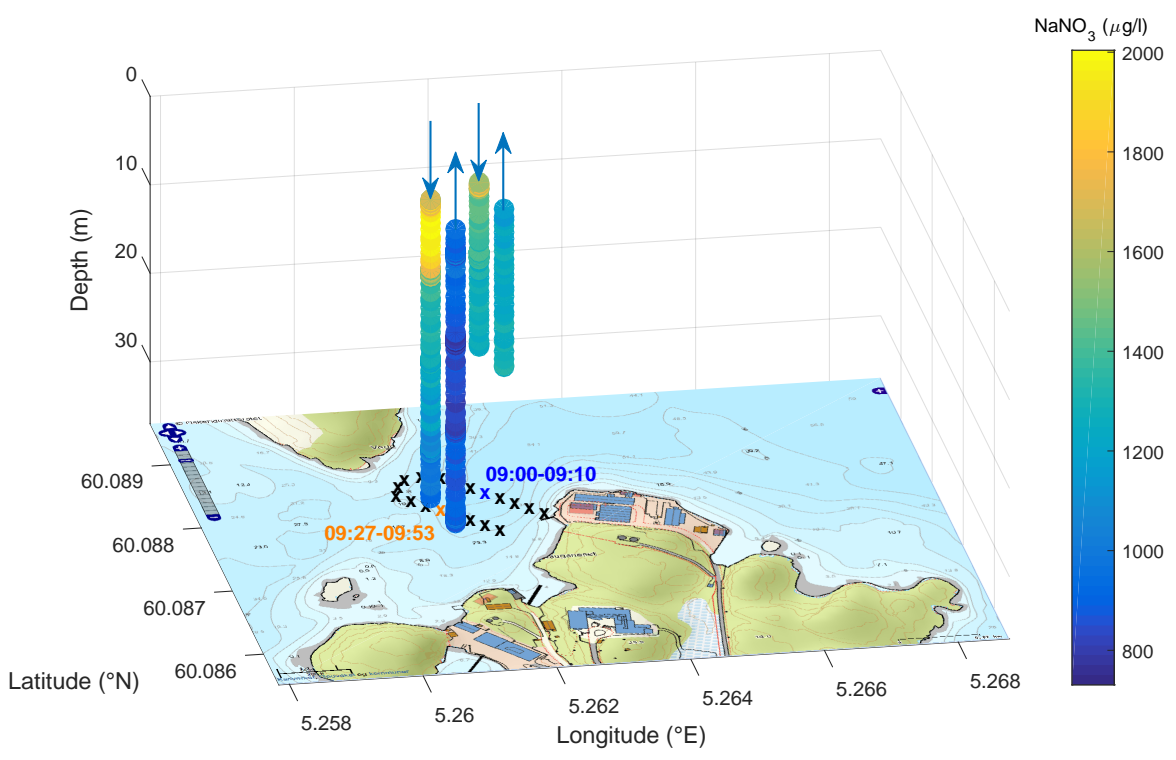

Figure 12. Defined mission plan and nitrate concentration for the two dive maneuvers during the mission in Austevoll on 31 October 2013. 


\subsection{Analysis of the Logged Data}

Figures 13 and 14 show the logged data of the depth, temperature, conductivity and oxygen for the two dive maneuvers. The gray shaded areas indicate the time ranges where the vehicle reached a new waypoint and holds a defined depth level for approximately $100 \mathrm{~s}$. The vertical speed (depth rate) was $0.1 \mathrm{~m} / \mathrm{s}$. The time lag of the temperature measurement is the result of the time constant of the temperature sensor $\left(\tau_{\text {Temperature }}<10 \mathrm{~s}[21,22]\right.$ ), the signal filtering and the insufficient incident flow of the sensors as a result of turbulences especially during the downward dives and hovering phases. (The time constant $\tau$ is derived from the step response of the first-order element as the duration of the time interval, required to reach $63.2 \%$ of the steady-state value of the step response [23]. The parameter settling time $T_{90}$ is often found in data sheets to describe the time characteristic of a sensor. This time is required to reach $90 \%$ of the steady-state value of the step response. In case of a first-order element and a negligible dead time in step response, the following relations to the time constant $\tau$ exist $T_{90}=2.3 \tau, T_{99}=4.6 \tau, T_{63}=\tau$.) It can be seen in Figure 14 that the conductivity around the fish farms increases with depth. This behavior is plausible because the minerals, such as salts from the feed, settle to lower depths. The oxygen level changes in Figure 14 correlate indirectly with the temperature. The oxygen concentration decreases with increasing water temperature, which is an important aspect for aquacultural production [24].
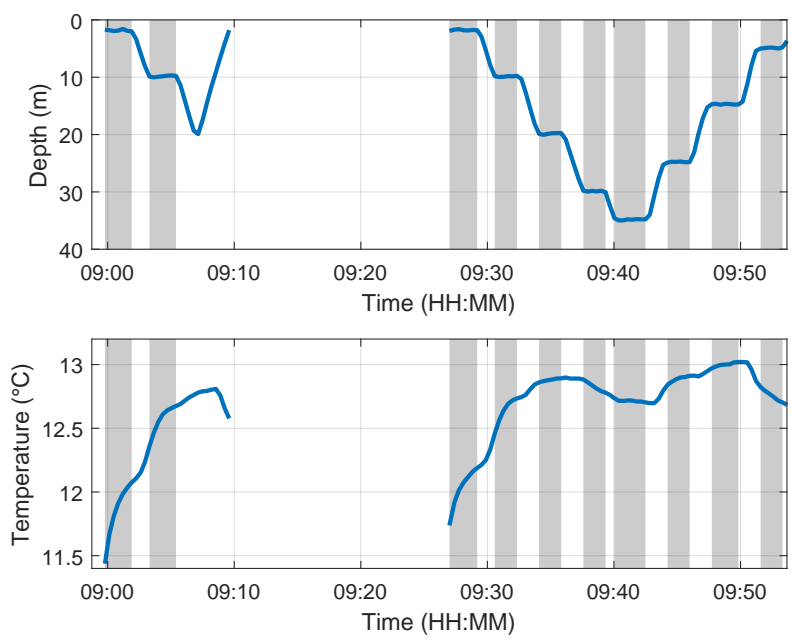

Figure 13. Depth and temperature data of the mission in Austevoll on 31 October 2013.
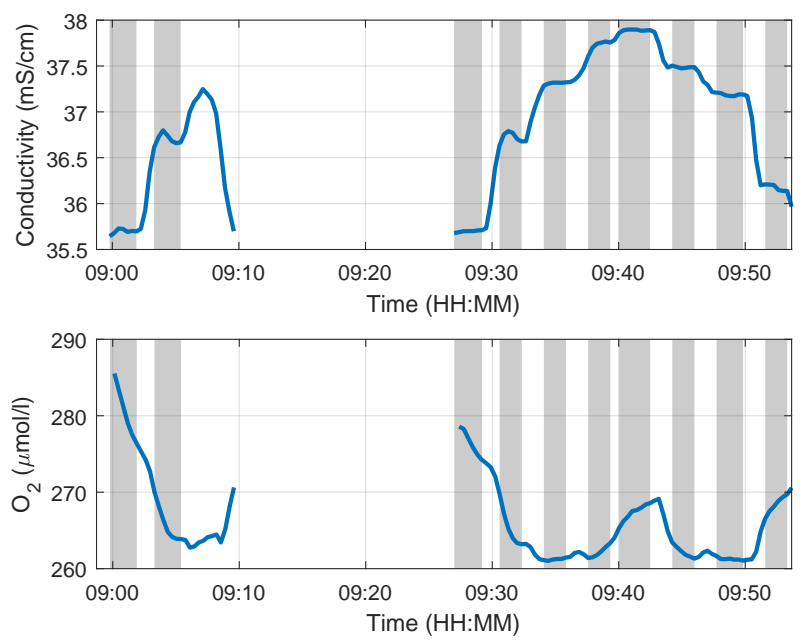

Figure 14. Conductivity and oxygen data of the mission in Austevoll on 31 October 2013. 
The data profiles of the two dives are shown in Figure 15. It can been seen that there is a difference between the downward and the upward dive profiles accented by the plateaus during the defined depth levels. One reason for this behavior is the large time delay in the temperature progression, as described previously. Since the temperature information will be used in the calculation for salinity and oxygen, these plateaus can also be found there. The influence of the time delay on the conductivity measurement $\left(\tau_{\text {Conductivity }}<1.3 \mathrm{~s}\right.$ [21]) is negligible, which can be seen in the similar conductivity progressions for the downward and upward dives. The calculation of the salinity with the method described in [25] provides the same curve progression as shown in Figure 14, which is the result of the internal sensor calculation. For the salinity calculation, the function sw_salt of the SeaWater MATLAB library from CSIRO [26] was used. The input variables of this function are conductivity, temperature and pressure. The large response time of the oxygen sensor $\left(\tau_{\text {Oxygen }}<25 \mathrm{~s}\right.$ [22]) is also responsible for the plateaus in the oxygen progressions.
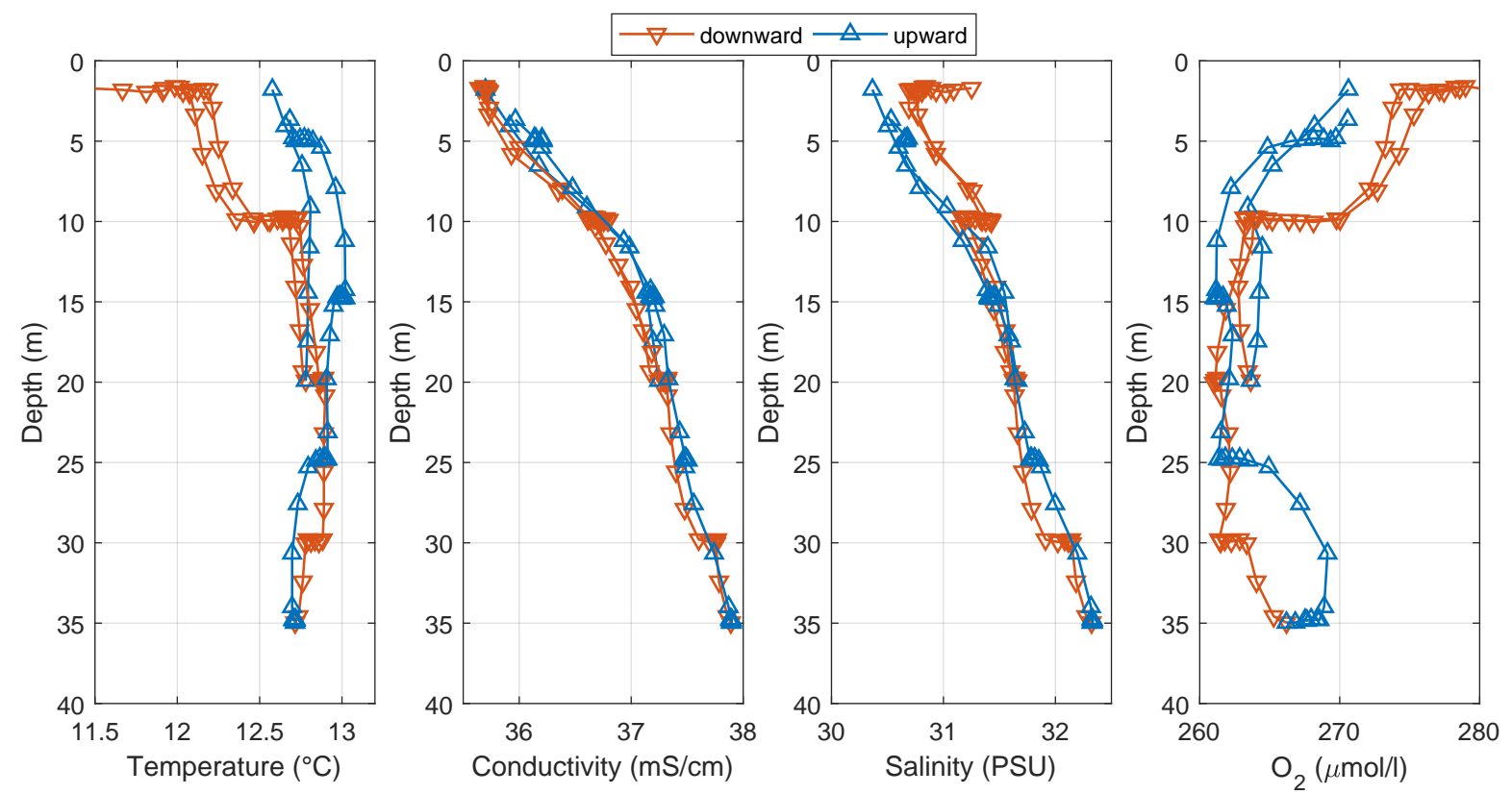

Figure 15. Data progressions of the mission in Austevoll on 31 October 2013.

The generated depth profiles using the mean of the data values after the settling process in the individual depths (right sides of the gray areas in Figures 13 and 14) are shown in Figure 16. Figure 17 shows the temperature and salinity profile from an ocean model of the Copernicus Marine Environment Monitoring Service (CMEMS) [27]. The principle profile shapes matched well with the logged profiles. The differences result from the inaccuracies of the model near the coast, the influence from the fish farms and the fresh water flow from local rivers.

Figure 12 shows the nitrate concentration as bars of the two dive profiles separated for the downward and upward process for a better illustration. The position and the time range of the dive maneuvers are marked with colored " $x$ " and font on the map. The high concentration of nitrate in the surface waters is a result of the discharge of nutritive substances from the fish farms and in flow from local rivers. The analysis of the ocean current data from CMEMS in the operation area during the trials shows an offshore current (east direction) of $0.12 \mathrm{~m} / \mathrm{s}$ up to $18 \mathrm{~m}$ depth and an onshore current (northwest direction) of $0.07 \mathrm{~m} / \mathrm{s}$ in greater depths. These current conditions are a reason for the concentration distribution. 

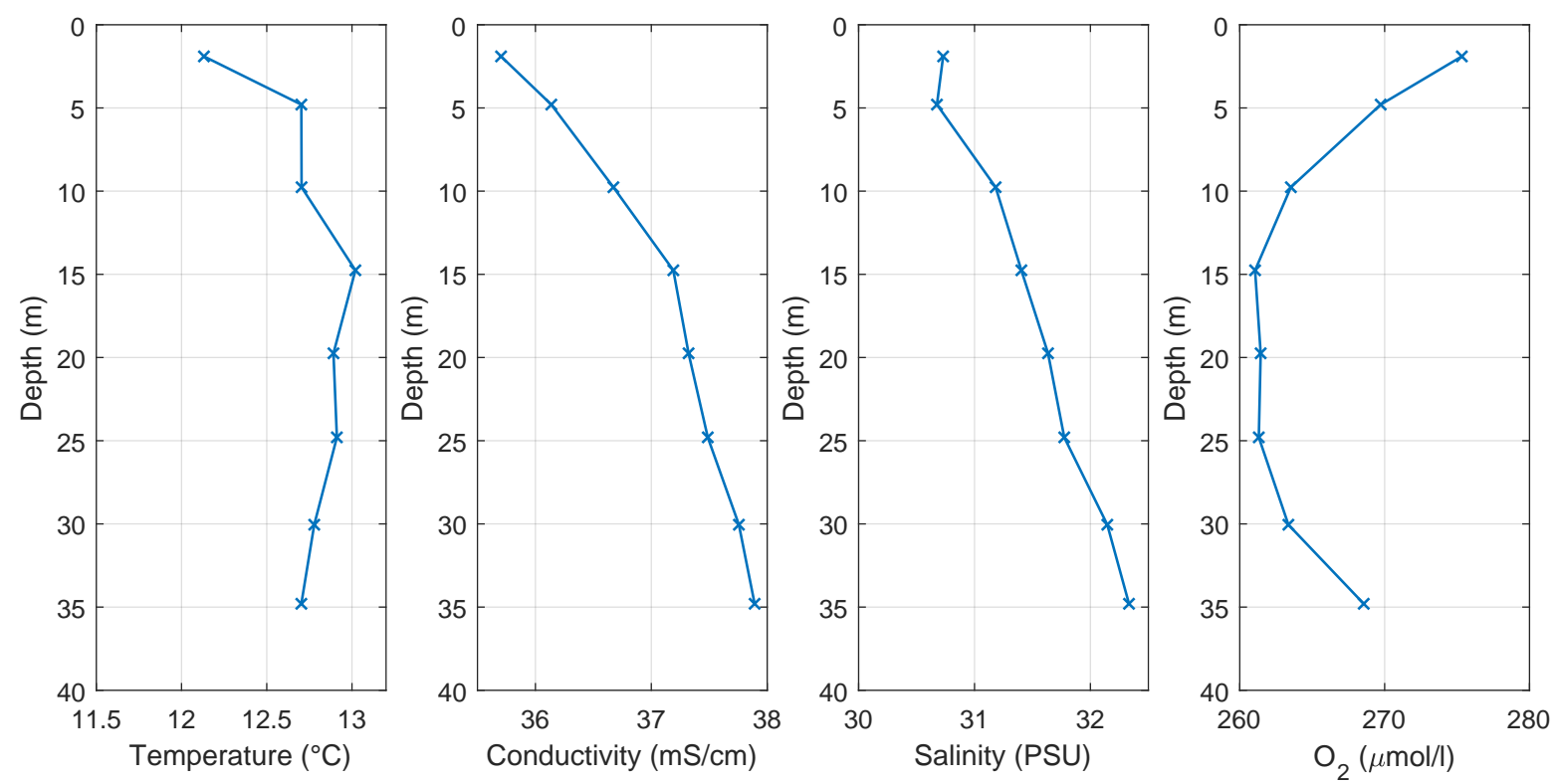

Figure 16. Data profiles of the mission in Austevoll on 31 October 2013.

Position: $60.083^{\circ} \mathrm{N} 5.250^{\circ} \mathrm{E}$ Date: $2013-10-3112: 00$
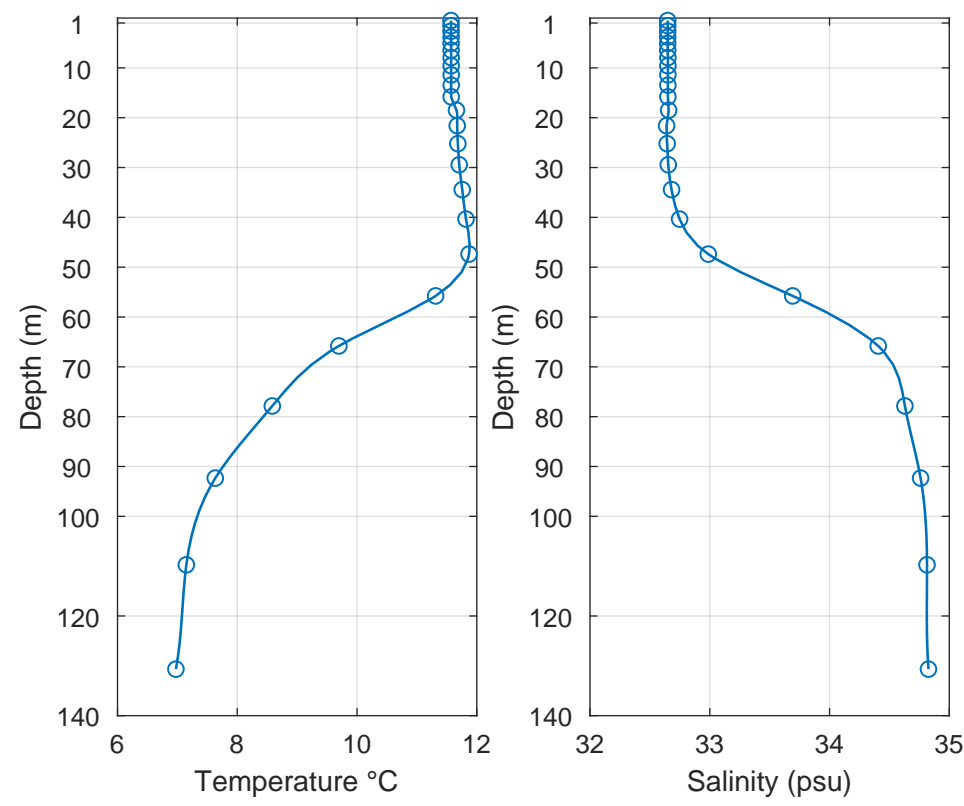

Figure 17. Temperature and Salinity Profile from the CMEMS model on 31 October 2013.

\section{Discussion}

\subsection{Gained Experience and Possible Improvements}

The trials in Norway show the possibilities and the limits of using an AUV for water quality analysis. The system allowed a fast analysis of the water quality parameters at every place around the fish farms. However, due to the heaviness of the AUV $(135 \mathrm{~kg})$, the launching process using the ramp or the crane was very challenging. Three employees were required for this. Here, a decrease of the vehicle-payload mass relation is desirable, which is currently 8.3 to 1 . Man-portable AUVs like a REMUS 100 (Hydroid, Inc., Pocasset, MA, USA) [28], an LAUV (OceanScan, Leça da Palmeira, Portugal) [29] or an Iver3 (L3 OceanServer , Fall River, MA, USA) [30] would have the ideal weight, 
but they cannot implement a $15 \mathrm{~kg}$ payload unit. This requires more powerful drive systems and additional battery units to work under real ocean current conditions. Likewise, a hover mode with the basic versions of these vehicles is not feasible. Furthermore, the definition of the mission plan and the initialization of the sensor system (flushing the measuring section, reference measurement of the spectrum of ultrapure water) required skilled personnel.

The communication via fiber optic cable, which was used during the trials, will not be used in future real operations. Two configurations are possible: (a) an acoustic link will provide low bandwidth communication for telemetry, online measurement data transfer and emergency situations; (b) a collision avoidance system will work on board, which is described in[31]. This system uses the information from the obstacle avoidance sonar at the front of the AUV (see Figure 1) and generates setpoint commands for the autopilot controllers in a collision situation. Previous projects have shown problems for a reliable detection of fishing nets and mooring lines by the sonar.

For future use, a simplification of the sensor system is necessary. This includes an automatic initialization process and a transparent depiction of the data. It is also conceivable to relocate the water-bearing sensor parts of the nitrate measurement outside of the hull segment. This leads to a smaller and more robust system as well as to an increase in operational safety because no sample water needs to be pumped into the vehicle. In addition, the correct incident flow of the sensors with sample water in hover mode has to be analyzed in detail. The time delay of the standard sensors was noticeable. In case of a mission consisting of saw tooth profiles, an additional correction algorithm or a sensor unit which has smaller response times installed in gliders [32] have to be used. The shipping traffic around the fish farms and the related high risk of collision with the AUV was underestimated at the beginning of the trials.

\subsection{Modular Sensor System}

The gained experience and the discussions with the employees of the fish farm show that an AUV is not the ultimate solution for fish farms. They need an easy-to-use system with a short lead and follow-up time. Based on these considerations, a modular sensor system will be favored (see Figure 18). This system has several extension levels with a growing degree of properties such as autonomy and operation range (see spider web diagram in Figure 18 (A spider web diagram allows the visualization of multiple qualitative variables in a two-dimensional chart. Every variable is represented by an axis. A high axis value corresponds with a good quality. By connecting several axis values with lines, a star figure is shown. The value of the resulting figure surface corresponds with the total quality.)). The base module includes the sensors, the battery and a computer unit covered in a pressure hull. The first level consists of a winch on the seabed and the sealed base module (see Figure 18 left). The sensor system can be lifted automatically or controlled by an operator. Because the park position of the sensor system is on the seabed, the likelihood of a collision or the obstruction of shipping traffic is very low. The disadvantage is the locally limited analysis of the water quality parameters in a water column. This can be done by the extension of the base module with actors and a cable-based or an acoustical communication system. This allows an expansion of the operation radius, which is restricted by the cable length or the maximum transmission range of the acoustic modem. Such a remotely operated vehicle (ROV) allows the online monitoring of the water quality parameters and the control of the vehicle during the mission. It is also possible to react to unforeseen events like ship traffic or concentration anomalies. An autonomous underwater vehicle (AUV) reaches the highest level of autonomy. This requires an exact navigation system on board and additional software and hardware modules to achieve an autonomous behavior (collision avoidance, condition monitoring). A mission plan with the defined tasks is sent to the vehicle before beginning a mission (see Section 2.2.2). Since an interaction with the vehicle is not possible during the mission, the logged data can be analyzed only after the mission. The usage of a mobile carrier platform required additional personnel and an expenditure for the preparation and follow-up of the mission. Intuitive handling in all autonomy levels is an important requirement of future use in fish farms. 


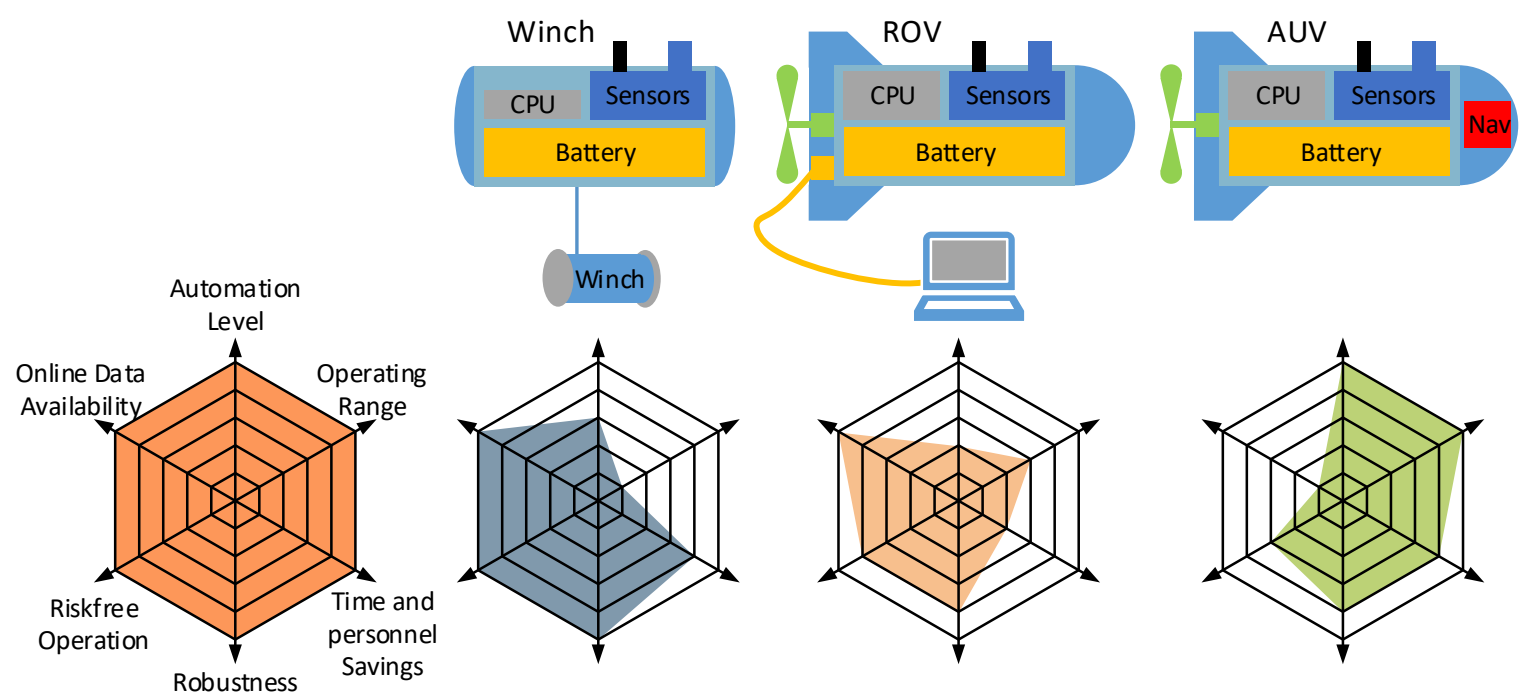

Figure 18. Modular sensor system with schematic and spider web diagrams for Winch, ROV and AUV.

\section{Conclusions}

In this paper, we present the concept, the technical implementation and the practical application of a miniaturized sensor system integrated into an autonomous underwater vehicle (AUV) for real time acquisition of water quality parameters around fish farms. We demonstrated the possibilities and the restrictions of using autonomous underwater vehicles for water quality analysis for aquaculture and coastal areas. Interested readers will find detailed information about the operating principle of the sensor system and the AUV. Furthermore, we discussed how important an intuitive and efficient handling of a modular sensor system is for future users.

Author Contributions: All authors discussed the results of the field trials and were involved in the revision process of the manuscript. M.E. was the group leader at Technische Universität Ilmenau and developed the interface and framework software in C++ for the SC. C.A. was the project manager of the SALMON project. M.J. designed and implemented the software for the CC. T.P. was responsible for the integration of the sensor system in the AUV. D.K. designed the navigation system. K.B. was the group leader of $4 \mathrm{HJE}$ and responsible for the requirement analysis and the design of the sensor system. M.B. has been in charge of the design of the nitrate sensor system. H.W. organized the field trials in Norway and defined the mission scenario.

Funding: This work was supported by the European Regional Development Fund (ERDF) of the European Union via the Thuringian Coordination Office TNA \# TNA VIII-1/2011.

Acknowledgments: Several colleagues and students of the Technische Universität Ilmenau were involved in this project. The authors thank Matthias Schneider for programming the menu-guided planning system and Ralf Taubert for designing the vehicle model and the autopilot as well as for his support during the field trials in Thuringia and Norway. We thank Samuel Gebhardt from $4 \mathrm{HJE}$ for assisting with the sensor system. Special thanks also go to the employees of the research station in Austevoll for their assistance during the field trials.

Conflicts of Interest: The authors declare no conflict of interest.

\section{Abbreviations}

The following abbreviations are used in this manuscript:

$\begin{array}{ll}\text { 4HJE } & -4 \mathrm{H}-\mathrm{JENA} \text { engineering GmbH } \\ \text { AUV } & \text { Autonomous Underwater Vehicle } \\ \text { CC } & \text { Control Computer } \\ \text { CMEMS } & \text { Marine Environment Monitoring Service } \\ \text { DVL } & \text { Doppler Velocity Log } \\ \text { GES } & \text { Good Environmental Status }\end{array}$




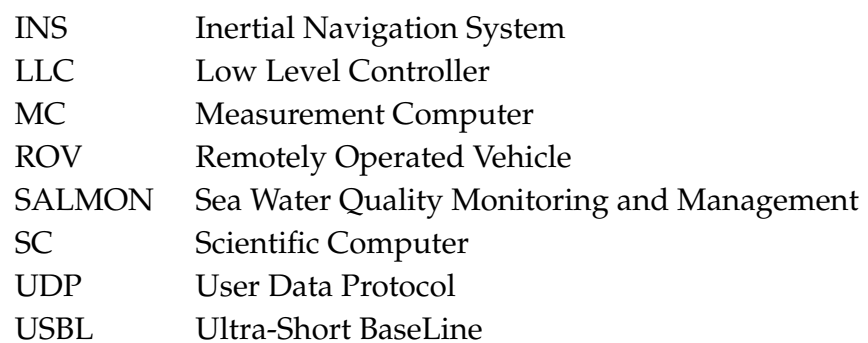

\section{References}

1. World Bank. Fish to 2030-Prospects for Fisheries and Aquaculture, 2013. Available online: http:/ / www.fao.org/docrep/019/i3640e/i3640e.pdf (accessed on 4 June 2018).

2. Kobayashi, M.; Msangi, S.; Batka, M.; Vannuccini, S.; Dey, M.M.; Anderson, J.L. Fish to 2030: The Role and Opportunity for Aquaculture. Aquac. Econ. Manag. 2015, 19, 282-300, doi:10.1080/13657305.2015.994240. [CrossRef]

3. European Union. COMMISSION DECISION (EU) 2017/848 of 17 May 2017 Laying down Criteria and Methodological Standards on Good Environmental Status of Marine Waters and Specifications and Standardised Methods for Monitoring and Assessment, and Repealing Decision 2010/477/EU, 2017. Available online: http:/ / eur-lex.europa.eu/legal-content/EN/TXT/PDF/?uri=CELEX:32017D0848\&from= EN (accessed on 16 April 2018).

4. Parra, L.; Sendra, S.; García, L.; Lloret, J. Design and Deployment of Low-Cost Sensors for Monitoring theWater Quality and Fish Behavior in Aquaculture Tanks during the Feeding Process. Sensors 2018, 18, 750, doi:10.3390/s18030750. [CrossRef] [PubMed]

5. Simbeye, D.; Yang, S.F. Water Quality Monitoring and Control for Aquaculture Based on Wireless Sensor Networks. J. Netw. 2014, 9, 840-849, doi:10.4304/jnw.9.4.840-849. [CrossRef]

6. Cario, G.; Casavola, A.; Gjanci, P.; Lupia, M.; Petrioli, C.; Spaccini, D. Long lasting underwater wireless sensors network for water quality monitoring in fish farms. In Proceedings of the OCEANS '17 IEEE, Aberdeen, Scotland, 19-22 June 2017; pp.1-6

7. Rundtop, P.; Frank, K. Experimental evaluation of hydroacoustic instruments for ROV navigation along aquaculture net pens. Aquac. Eng. 2016, 74, 143-156, doi:10.1016/j.aquaeng.2016.08.002. [CrossRef]

8. Duda, A.; Schwendner, J.; Stahl, A.; Rundtop, P. Visual Pose Estimation for Autonomous Inspection of Fish Pens. In Proceedings of the OCEANS'15 MTS/IEEE, Genova, Italy, 18-21 May 2015.

9. Borović, B.; Vasilijević, A.; Kuljača, O. Potentials of Using Underwater Robotics for Fishing and Fish Farming. In Proceedings of the 10th International Workshop Methods for the Development and Evaluation of Maritime Technologies-DEMaT'11, Split, Croatia, 21-23 June 2011.

10. Takagi, M.; Mori, H.; Yimit, A.; Hagihara, Y.; Miyoshi, T. Development of a Small Size Underwater Robot for Observing Fisheries Resources-Underwater Robot for Assisting Abalone Fishing. J. Robot. Mechatron. 2016, 28, 397-403. [CrossRef]

11. Eichhorn, M.; Taubert, R.; Ament, C.; Jacobi, M.; Pfuetzenreuter, T. Modular AUV System for Sea Water Quality Monitoring and Management. In Proceedings of the OCEANS '13 MTS/IEEE, Bergen, Norway, 10-13 June 2013.

12. Taubert, R.; Eichhorn, M.; Ament, C.; Jacobi, M.; Karimanzira, D.; Pfuetzenreuter, T. Model Identification and Controller Parameter Optimization for an Autopilot Design for Autonomous Underwater Vehicles. In Proceedings of the OCEANS '14 MTS/IEEE, Taipei, Taiwan, 7-10 April 2014.

13. Karimanzira, D.; Jacobi, M.; Pfuetzenreuter, T.; Rauschenbach, T.; Eichhorn, M.; Taubert, R.; Ament, C. First testing of an AUV mission planning and guidance system for water quality monitoring and fish behavior observation in net cage fish farming. Information Processing in Agriculture. Inf. Process. Agric. 2014, 1, 131-140, doi:10.1016/j.inpa.2014.12.001. [CrossRef]

14. -4H-JENA engineering GmbH. From the Sea Floor to the Stars-Customized Technology. Available online: http:/ / www.4h-jena.de/en/start (accessed on 16 April 2018).

15. Sea-Bird Scientific. SUNA V2 Datasheet. Available online: http://www.seabird.com/sites/default/files / documents / datasheet_SUNAV2_0.pdf (accessed on 18 April 2018). 
16. Sea-Bird Scientific. ISUS V3 Datasheet. Available online: http://www.seabird.com/sites/default/files/ documents/datasheet_isusv3.pdf (accessed on 18 April 2018).

17. MacIntyre, G.; Plache, B.; Lewis, M.R.; Andrea, J.; Feener, S.; McLean, S.D.; Johnson, K.S.; Coletti, L.J.; Jannasch, H.W. ISUS/SUNA nitrate measurements in networked ocean observing systems. In Proceedings of the Oceans 2009 MTS/IEEE, Biloxi, MS, USA, 26-29 October 2009.

18. Pfuetzenreuter, T.; Renkewitz, H. ConSys-A new software framework for underwater vehicles. In Proceedings of the OCEANS '10 IEEE, Sydney, Australia, 24-27 May 2010.

19. Kalwa, J. The GREX-Project: Coordination and control of cooperating heterogeneous unmanned systems in uncertain environments. In Proceedings of the OCEANS '09 IEEE, Bremen, Germany, 11-14 May 2009.

20. Simonetti, P.J. Slocum Glider, Design and 1991 Field Trials; Technical report; Webb Research Corporation, North Falmouth, MA, USA, 1992.

21. Aanderaa Data Instruments AS. Data \& Specification Sheets, Conductivity Sensor 3919. Available online: http:/ / www.aanderaa.com/media/pdfs/Conductivity-Sensor-3919.pdf (accessed on 16 April 2018).

22. Aanderaa Data Instruments AS. Data \& Specification Sheets, Oxygen Sensor 3835. Available online: http: / / www.aanderaa.com/media/pdfs/Oxygen-Optode-3835-4130-4175.pdf (accessed on 16 April 2018).

23. IEC. International electrotechnical vocabulary—Part 351: Control technology (IEC 60050-351:2013), 2014. Available online: http:/ / www.electropedia.org/iev /iev.nsf/display?openform\&ievref=351-45-32 (accessed on 4 June 2018).

24. Kitani, O. CIGR Handbook of Agricultural Engineering: Animal Production and Aquacultural Engineering; American Society of Agricultural Engineers: St. Joseph, MI, USA, 1999; Volume 2.

25. Fofonoff, N.M.R., Jr. Algorithms for Computation of Fundamental Properties of Seawater; Technical Report; UNESCO: Paris, France, 1983.

26. CSIRO. SeaWater MATLAB Library. Available online: http://www.cmar.csiro.au/datacentre/ext_docs / seawater.htm (accessed on 16 April 2018).

27. Copernicus Marine Environment Monitoring Service. Providing Products and Services for All Marine Applications. Available online: http:/ / marine.copernicus.eu/ (accessed on 16 April 2018).

28. Hydroid Inc.. Remus 100 Autonomous Underwater Vehicle. Available online: https://www.hydroid.com/ sites/default/files/product_pages/New_Generation_REMUS_100_\{\%\}20Brochure_2017_1.pdf (accessed on 16 April 2018).

29. OceanScan. Light Autonomous Underwater Vehicle. Available online: http://www.oceanscan-mst.com/ light-autonomous-underwater-vehicle/\#vehicle-specifications (accessed on 16 April 2018).

30. L3 OceanServer. Affordable Work Class Autonomous Underwater Vehicle (AUV). Available online: http:/ / www.iver-auv.com/AP_003_Iver3.pdf (accessed on 16 April 2018).

31. Eichhorn, M.. A reactive obstacle avoidance system for an autonomous underwater vehicle. In Proceedings of the 16th IFAC world congress. Prague, Czech Republic, 3-8 July 2005; pp. 331-336.

32. Sea-Bird Scientific. Glider Payload CTD (Optional DO). Available online: http://www.seabird.com/sites / default/files/documents/GliderPayloadCTDBrochureAug15.pdf (accessed on 22 April 2018).

(c) 2018 by the authors. Licensee MDPI, Basel, Switzerland. This article is an open access article distributed under the terms and conditions of the Creative Commons Attribution (CC BY) license (http:/ / creativecommons.org/licenses/by/4.0/). 